\title{
New Drugs for Acute Heart Failure
}

\author{
MOHAMMAD SALMAN ${ }^{1}$, SYEDALIAHSAN ${ }^{2}$, MANZOOR MAHMOOD ${ }^{2}$, MD. KHAIRUL ANAM ${ }^{2}$, SHAHED MOHAMMAD \\ ANWAR $^{3}$, ROKSANAAFROSE $^{4}$, MD. ABU SIDDIQUE ${ }^{2}$, MD. ASHRAF UDDIN SULTAN $^{2}$, FAZLUR RAHMAN $^{2}$ \\ ${ }^{1}$ Department of Cardiology, Anwer Khan Modern Medical College, Dhanmondi, Dhaka, ${ }^{2}$ Department of Cardiology, \\ Bangabandhu Sheikh Mujib Medical University, Dhaka, ${ }^{3}$ University of Science and Technology, Chittagong, ${ }^{4}$ Chittagong Ma O \\ Shishu and General Hospital, Chittagong. \\ Address for Correspondence: Dr. Md. Salman, Department of Cardiology, Anwar Khan Modern Medical College, Dhaka, \\ E-mail: drmdsalman@gmail.com
}

\begin{abstract}
Acute heart failure is a major health problem responsible for several million hospitalizations worldwide each year. Standard therapy has not changed for long time and includes diuretics and variable use of vasodilators or inotropes. Recently Nesiritide and Levosimendan are two drugs for the treatment of acute heart failure which have been approved by the Food and Drug Administration (FDA) and European Medicines Agency (EMEA), respectively. There was little concern that Nesiritide can worsen the renal failure but recent trials had abolished this concern.
\end{abstract}

Key Words: Acute heart failure, Nesiritide, Levosimendan.

Acute heart failure (AHF) is defined as rapid change in heart failure signs and symptoms resulting in the need for urgent therapy. The syndrome is complex and has multiple etiologies. ${ }^{1}$ Most of the therapies for acute heart failure are mainly addressing the symptomatic relief of fluid overload and only very few effective treatments are available for AHF that improves clinical outcomes.

There are three groups of patients with acute heart failure on the basis of systolic blood pressure (SBP) at the time of presentation. ${ }^{2,3}$

1. Patients presenting with hypertension and have preserved systolic function. Their in-hospital mortality is approximately $2 \%$.

2. Patients presenting with normal blood pressure tend to have a lower left ventricular ejection fraction (LVEF) and signs and symptoms of pulmonary/systemic congestion (edema) before and at the time of presenaion. The in-hospital mortality is approximately $3 \%$.

3. Patients presenting with low blood pressure generally have a low LVEF, and have a previous history of HF. In-hospital mortality is approximately $7 \%$.

These three patient groups not only differ prognostically but also require appropriately tailored pharmacologic treatments. Most patients with acute heart failure with high or normal blood pressure at the time of admission present with pulmonary or systemic congestion and relatively normal cardiac output, and their early management is mainly directed at correcting high LV filling pressure and after load. Conversely, in patients hospitalized with acute heart failure who present with low SBP, first-line therapies are targeted at low cardiac output in addition to congestion. Most of the drugs commonly used for the treatment of acute heart failure having wellknown limitations and have been associated with an early increase in the risk of death. ${ }^{4-8}$ Therefore, there is an immense need for new drugs for the acute heart failure patients presenting with congestion or low cardiac output that can safely improve hemodynamics, symptoms and possibly long-term survival. The aim of this paper is therefore to review recent advances on emerging drugs in acute heart failure and to summarize evidence of clinical benefit.

\section{Recently approved agents for acute heart failure}

Early treatment of patients admitted with acute heart failure is critical because the prompt use of pharmacologic agents may affect long-term morbidity and mortality. There are very little scope for assessing new drugs, and re-evaluating clinical trial design. Actually the most of the studies evaluate small to moderate short-term hemodynamic or symptom-focused outcome.

One problem is note worthy that research in acute heart failure is, conducted during the hospitalization phase of patients, when often symptoms such as dyspnea are markedly reduced.

Nesiritide and Levosimendan are two drugs for the treatment of acute heart failure which have been approved by the Food and Drug Administration (FDA) and European Medicines Agency (EMEA), respectively. However, the 
safety and efficacy of these agents have recently been questioned and Nesiritide has not been approved by EMEA and Levosimendan has not been approved by FDA. Although previous studies demonstrated that these new therapies improve hemodynamic parameters, recent metaanalyses and randomized trials suggest that they may increase or have comparable impact on long-term mortality, compared to conventional drugs.

\section{Nesiritide}

Nesiritide is a recombinant form of human brain natriuretic peptide (BNP) that exerts vasodilatory effects on arterial, venous, and coronary vessels, leading to increased cardiac output. Studies of Nesiritide in the treatment of acute heart failure have documented beneficial effects on hemodynamics (reductions in PCWP and systemic vascular resistance and increases in cardiac output) and symptoms. ${ }^{9,10}$ The Vasodilatation in the Management of Acute Congestive Heart Failure (VMAC) trial was a multicenter, randomized, double-blind, controlled trial designed to compare the hemodynamic and clinical effects and safety of intravenous Nesiritide and intravenous Nitroglycerin added to standard care in patients hospitalized for dyspnea at rest due to acute heart failure. ${ }^{11}$ Patients were randomized to receive Nesiritide, Nitroglycerin or placebo for 3 hours. After 3 hours, Nesiritide reduced PCWP to a significantly greater degree than did nitroglycerin or placebo. Nesiritide significantly improved dyspnea compared with placebo but resulted in no significantly different improvement in dyspnea compared with nitroglycerin. After 24 hours, the mean reduction in PCWP was significantly greater in the Nesiritide group than in the nitroglycerin group, but there was no significant difference in dyspnea between the two groups. Adverse events (most commonly headache) occurred significantly less frequently with Nesiritide than with Nitroglycerin. There was no significant difference in 6-month mortality rates in the Nesiritide group compared with the Nitroglycerin group.

The safety and efficacy of Nesiritide has been questioned recently. ${ }^{12}$ The main concern is about the possible adverse effects of Nesiritide therapy on renal function ${ }^{13}$ and shortterm mortality, in comparison with standard diuretic and vasodilator therapies. ${ }^{14,15}$ The BNP-CARDS (B-Type Natriuretic Peptide in Cardiorenal Decompensation Syndrome) trial randomized 75 consecutive patients with acute heart failure and baseline renal dysfunction to receive Nesiritide ( 0.01 ìg/kg/min with or without a 2-ìg/kg bolus) or placebo (5\% dextrose in water) for $48 \mathrm{~h}$ in addition to usual care. ${ }^{16}$ There were no significant differences in the increase in serum creatinine by $20 \%$ and change in serum creatinine between the two groups. In addition, there were no significant differences in the secondary end points of change in weight, intravenous frusemide use, discontinuation of the infusion due to hypotension, or 30-day death or hospital readmission. ${ }^{16}$ A possible explanation for the disparate findings between this and previous studies is the use of a bolus dose. Probably Nesiritide has some effect on glomerular filtration rate due to the significant hypotension occurring with the bolus dose, possibly accounting for some of the worsened renal function seen in other retrospective analyses. Another possible difference between the results of this trial and previous observations are the timing of the Nesiritide infusion initiation and the dose of infusion used. Data from the second Follow-Up Serial Infusions of Nesiritide in Advanced Heart Failure (FUSION-2) trial have been presented. ${ }^{17}$ The trial randomized 911 patients to receive Nesiritide as a 2-ìg/kg bolus followed by a 0.01-ìg/kg/min infusion for 4 to $6 \mathrm{~h}$ or a placebo regimen, once or twice a week for 12 weeks. Inclusion in the trial required being in NYHA class III or IV with an LVEF d" $40 \%$ and a history of at least two prior hospitalizations for acute heart failure within the past year. Patients in NYHA class III were only recruited if their creatinine clearance was e" $60 \mathrm{~mL} / \mathrm{min}$. No outpatient IV inotropic or vasodilator therapy was allowed during the study. At the end of the study, there were no significant differences in rates of the primary end point of all cause mortality or cardiovascular or cardio renal hospitalization or in rates of its individual component events. ${ }^{17}$ The Acute Study of Clinical Effectiveness of Nesiritide in Subjects With Decompensated Heart Failure (ASCEND-HF) trial is randomized, double-blind, placebocontrolled study. The following conclusions came from this study. Nesiritide did not reduce the rate of recurrent heart failure hospitalization or death at 30 days. Nesiritide reduced dyspnea to a modest degree, consistent with previous findings but did not meet pre-specified protocol criteria for statistical significance at 6 and 24 hours. Nesiritide did not affect 30-day all cause mortality nor did it worsen renal function as had been suggested by prior meta-analyses of smaller studies. Nesiritide can now be considered as a safe therapy in patients with acute heart failure.

\section{Levosimendan}

Calcium sensitizing agents are a newer class of positive inotropic drugs that include levosimendan, pimobendan, senazodan, EMD-53998, and its enantiomer, ED- 57033. ${ }^{18}$ These drugs exert a dose-dependent calcium sensitizing 
mechanical enhancement on the failing heart via various type of biochemical mechanisms, including the enhancement of troponin- $\mathrm{C}$ affinity for calcium, the direct stabilization of the calcium-induced conformation of troponin-C, or the action distal to the troponin- $C$ molecule. ${ }^{18}$ However, some molecules such as pimobendan, EMD- 53398, senazodan and possibly levosimendan may act as phosphodiesterase inhibitors at therapeutic doses, causing a deleterious increase of intracellular cyclic AMP. This effect seems to be an essential limitation for the use of these drugs clinically. ${ }^{18}$ Levosimendan is the most studied calcium sensitizer and has recently been introduced in many countries for the treatment of acute heart failure. Levosimendan acts via two complementary mechanisms. ${ }^{19}$ It enhances contractility by improving cardiac myofilament response to intracellular calcium and it reduces the cardiac workload by opening ATP-dependent potassium channels for dilation of blood vessels. ${ }^{19}$ Indeed levosimendan-induced decrease in right and left ventricular after load may be beneficial in failing hearts. ${ }^{19,20}$ Furthermore, levosimendan differs from classic inotropes because of its ability to improve myocardial efficiency without increasing myocardial oxygen demand, its effects on coronary blood flow, and its lack of negative lusitropic effects. ${ }^{19,20}$ Data from several trials suggest that levosimendan appears to improve hemodynamics, symptoms and neurohormonal response $\mathrm{e}^{21-23}$ in acute heart failure and to possibly prolong survival in some subsets of patients. For instance, in the Randomized Study on Safety and Effectiveness of Levosimendan in Patients with Left Ventricular Failure Due to an Acute Myocardial Infarct (RUSSLAN) study ${ }^{24}$ and in the Levosimendan Infusion Versus Dobutamine (LIDO) study $^{25}$ levosimendan was associated with hemodynamic improvements and in secondary with a lower risk of death compared to Dobutamine and in post-MI or low-output heart failure patients. The recent REVIVE-1 and -2 (Randomized Evaluations of Levosimendan) and SURVIVE (Survival of Patients with Acute Heart Failure in Need of Intravenous Inotropic Support) trials showed that levosimendan was superior to placebo or Dobutamine, respectively, in producing clinical improvement and beneficial neurohormonal modulation (as expressed by the reduction in plasma BNP) in patients with acute heart failure. ${ }^{26-28}$ However, levosimendan failed to lead in a reduction of in hospital and 6-month mortality compared with Dobutamine (SURVIVE: primary end point) in these patients. More specifically, in the REVIVE-2 study, ${ }^{26}$ 90day all-cause mortality was $15.1 \%$ in the levosimendan group and $11.6 \%$ among placebo-treated patients $(p=0.210)$; this numerical increase in deaths in the levosimendan group was associated with the higher incidence of hypotensive episodes than in the placebo group. The SURVIVE trial ${ }^{28}$ randomized 1327 patients with acute diastolic heart failure and a left ventricular ejection fraction of $30 \%$ or less, who required intravenous inotropic therapy because of insufficient response to intravenous diuretics or vasodilators. All patients received standard treatment and were randomized to the addition of either a 12-ìg/kg bolus of levosimendan followed by a stepped dose regimen of $0.1-0.2 \mathrm{ig} / \mathrm{kg} / \mathrm{min}$ infusion for a maximum of 24 hours or Dobutamine at a dose of at least $5 \mathrm{ig} / \mathrm{kg} / \mathrm{min}$ for at least 24 hours. The primary end point in the SURVIVE trial (all-cause mortality at 6 months) showed similar results for both levosimendan and Dobutamine (26.2\% and 27.9\%, respectively; $\mathrm{p}=0.401$ )

Interestingly SURVIVE shows that levosimendan induced a much greater decrease in BNP compared to Dobutamine, over the first week of treatment. ${ }^{28}$ Levosimendan is currently in clinical use in several countries (excluding US) and is indicated in patients with symptomatic low cardiac output HF secondary to cardiac systolic dysfunction without severe hypotension. ${ }^{29}$ Further studies are clearly needed in order to identify proper dosages and timing of infusion, and the subset of patients who may benefit more from this drug.

\section{Conclusion}

No new agent has demonstrated a clear benefit in terms of long-term clinical outcomes compared to conventional therapies. Since recent studies demonstrated that early management may influence long-term outcomes, a major challenge in acute heart failure trials remains the development of appropriate time for initiation of therapy and end points for evaluating the efficacy of these new pharmacologic therapies.

\section{References}

1. Gheorghiade M, Zannad F, Sopko G, et al: Acute heart failure syndromes: Current state and framework for future research. Circulation 2005; 112:3958-68

2. Gheorghiade M, Abraham W, Albert N, et al. Systolic blood pressure at admission, clinical characteristics, and outcomes in patients hospitalized with acute heart failure. JAMA 2006;296:2217-26.

3. Filippatos G, Zannad F. An introduction to acute heart failure syndromes: definition and classification. Heart Fail Rev 2007;12:87-90.

4. Shin DD, Brandimarte F, De Luca L, et al. Review of current and investigational pharmacologic agents for acute heart failure syndromes. Am J Cardiol 2007; 99: 4A-23A. 
5. Bayram M, De Luca L, Massie MB, Gheorghiade M. Reassessment of dobutamine, dopamine, and milrinone in the management of acute heart failure syndromes. Am J Cardiol 2005; 96: 47-58.

6. Cotter G, Metzkor E, Kaluski E, et al. Randomised trial of high-dose isosorbide dinitrate plus low-dose furosemide versus high-dose furosemide plus low-dose isosorbide dinitrate in severe pulmonary oedema. Lancet 1998;351:389-93.

7. Hasselblad V, Stough WG, Shah MR, et al. Relation between diuretic dose and outcome in a heart failure population: results of the ESCAPE trial [abstract]. J Card Fail 2005;11:S157.

8. Cohn JN, Franciosa JA, Francis GS, et al. Effect of shortterm infusion of sodium nitroprusside on mortality rate in acute myocardial infarction complicated by left ventricular failure: results of a Veterans Administration cooperative study. N Engl J Med 1982;306:1129-35.

9. Mills RM, LeJemtel TH, Horton DP, et al. Sustained hemodynamic effects of an infusion of nesiritide (human btype natriuretic peptide) in heart failure: a randomized, doubleblind, placebo-controlled clinical trial. J Am Coll Cardiol 1999; 34: 155-62.

10. Colucci WS, Elkayam U, Horton DP, et al. Intravenous nesiritide, a natriuretic peptide, in the treatment of decompensated congestive heart failure. N Engl J Med 2000;343:246-53.

11. Publication Committee for the VMAC [Vasodilatation in the Management of Acute CHF] Investigators. Intravenous nesiritide vs nitroglycerin for treatment of decompensated congestive heart failure: a randomized controlled trial. JAMA 2002;287:1531-40.

12. Topol EJ. Nesiritide-not verified. $N$ Engl J Med 2005;353:113-6.

13. Sackner-Bernstein JD, Skopicki HA, Aaronson KD. Risk of worsening renal function with nesiritide in patients with acutely decompensated heart failure. Circulation 2005;111:1487-91.

14. Sackner-Bernstein JD, Kowalski M, Fox M, Aaronson K. Shortterm risk of death after treatment with nesiritide for decompensated heart failure: a pooled analysis of randomized controlled trials. JAMA 2005;293:1900-2005.

15. Aaronson KD, Sackner-Bernstein JD. Risk of death associated with nesiritide in patients with acutely decompensated heart failure [research letter]. JAMA 2006; 296: 1465-66.

16. Witteles RM, Kao D, Christopherson D, et al. Impact of nesiritide on renal function in patients with acute decompensated heart failure and pre-existing renal dysfunction a randomized, double-blind, placebocontrolled clinical trial. J Am Coll Cardiol 2007;50:1835-40.

17. Cleland JG, Coletta AP, Clark AL. Clinical trials update from the American College of Cardiology 2007: Alpha, Everest, Fusion II, Validd, Parr-2, Remodel, Spice, Courage, Coach,
Remadhe, pro-BNP for the evaluation of dyspnoea and THISdiet. Eur J Heart Fail 2007;9:740-45.

18. Parissis JT, Farmakis D, Nieminen M. Classical inotropes and new cardiac enhancers. Heart Fail Rev 2007;12:149-56.

19. De Luca L, Colucci WS, Nieminen MS, Massie BM, Gheorghiade M. Evidence-based use of levosimendan in different clinical settings. Eur Heart J 2006;27:1908-20.

20. Parissis J, Filippatos G, Farmakis D, Adamopoulos S, Paraskevaidis I, Kremastinos D. Levosimendan for the treatment of acute heart failure syndromes. Exp Opin Pharmacother 2005; 6(15): 2741-51.

21. Nieminen MS, Akkila J, Hasenfuss G, et al. Hemodynamic and neurohumoral effects of continuous infusion of levosimendan in patients with congestive heart failure. J Am Coll Cardiol 2000; 36: 1903-12.

22. Slawsky MT, Colucci WS, Gottlieb SS, et al. Acute hemodynamic and clinical effects of levosimendan in patients with severe heart failure. Circulation 2000; 102: 2222-27.

23. Parissis J, Panou F, Famakis D, et al. Effects of levosimendan on markers of left ventricular diastolic function and neurohormonal activation in patients with advanced heart failure. Am J Cardiol 2005; 96: 423-26.

24. MoiseyevVS, Poder P,AndrejevsN, et al. Safety and efficacy of a novel calcium sensitizer, levosimendan, in patients with left ventricular failure due to an acute myocardial infarction: a randomized, placebocontrolled, double-blind study (RUSSLAN). Eur Heart J 2002;23:1422-32.

25. Follath F, Cleland JG, Just H, et al. Efficacy and safety of intravenous levosimendan compared with dobutamine in severe low-output heart failure (the LIDO study): a randomized double-blind trial. Lancet 2002;360:196-202.

26. Packer M, Colucci WS, Fisher L. Development of a comprehensivenew endpoint for the evaluation of new treatments for acute decompensated heart failure: results with levosimendan in the REVIVE-1 study (Abstract). J Card Fail 2003;9:S61.

27. Packer M. The Randomized multicenter EValuation of Intravenous leVosimendan Efficacy-2 (REVIVE-2) trial. Latebreaking ClinicalTrials. Dallas, TX: American Heart Association, Annual ScientificSession; November 2005. p. $13-16$.

28. Mebazaa A, Nieminen MS, Packer M, et al. Levosimendan vsdobutamine for patients with acute decompensated heart failure: the SURVIVE Randomized Trial. JAMA 2007; 297: 1883-91.

29. Nieminen MS, Bohm M, Cowie MR, et al. Executive summary of the guidelines on the diagnosis and treatment of acute heart failure. The task force on acute heart failure of the European Society of Cardiology. Eur Heart J 2005; 26: 384416. 